\title{
Outcome of Pediatric Emergency Point-of-Care Ultrasound (POCUS) for Management of Skin and Soft Tissue Infection Prior to Ultrasound Program Implementation
}

\author{
Whitney Phillips, $\mathrm{BS}^{1}$, Benjamin Nti, MD, $\mathrm{MSc}^{2}$ \\ ${ }^{1}$ Indiana University School of Medicine, ${ }^{2}$ Indiana University School of Medicine, \\ Department of Emergency Medicine, Riley Hospital for Children, Indiana \\ University Health
}

Background and Hypothesis: Point-of-care ultrasound (POCUS) is underutilized for evaluation of skin and soft tissue infections (SSTI) in the pediatric emergency department (ED). This study seeks to determine the utilization of POCUS compared to formal radiology ultrasound for SSTI in the pediatric ED prior to an ultrasound program implementation. We hypothesize that POCUS utilization will be low but can lead to a decreased length of stay (LOS) and cost for patients with SSTI.

Experimental Design or Project Methods: This is a retrospective EMR chart review covering pre-implementation (July 2016-June 2017) and postimplementation (July 2018-June 2019) of a POCUS program curriculum. Patients ( $<18$ years old) were included based on screening for diagnoses via the international classification of diseases $9^{\text {th }}$ and $10^{\text {th }}$ revision codes for abscesses and cellulitis. We excluded patients who required admission and subspecialty consult or had other non-SSTI evaluation.

Results: Pre-Implementation period included 160 patients who met inclusion and exclusion criteria. Of these, $16(10 \%)$ received POCUS evaluation and $8(5 \%)$ received a radiology image evaluation. The majority of patients had cellulitis (80\%) when compared to abscess (20\%). The average LOS for POCUS ultrasound was 173 minutes compared to 304 minutes for radiology evaluation. The total cost for visit was $\$ 3,503$ for patients evaluated by POCUS compared to $\$ 8,875.56$ for patients who received radiology imaging.

Conclusion and Potential Impact: Taken together, the pre-implementation assessment of POCUS utilization in the pediatric emergency department was low but associated with decreased LOS and lower total ED cost when applied to SSTI management. 